\title{
Vulnerable atherosclerotic plaque detection by resonance Raman spectroscopy
}

Cheng-hui Liu

Susie Boydston-White

Arel Weisberg

Wubao Wang

Laura A. Sordillo

Adler Perotte

Vincent P. Tomaselli

Peter P. Sordillo

Zhe Pei

Lingyan Shi

Robert R. Alfano 


\title{
Vulnerable atherosclerotic plaque detection by resonance Raman spectroscopy
}

\author{
Cheng-hui Liu, ${ }^{\text {a }}$ Susie Boydston-White, ${ }^{\mathrm{b}}$ Arel Weisberg, ${ }^{\mathrm{c}}$ Wubao Wang, ${ }^{\mathrm{a}}$ Laura A. Sordillo, ${ }^{\mathrm{a}}$ Adler Perotte, ${ }^{\mathrm{d}}$ \\ Vincent P. Tomaselli, ${ }^{d}$ Peter P. Sordillo, ${ }^{a}$ Zhe Pei, ${ }^{a}$ Lingyan Shi, ${ }^{a}, \mathrm{e}$ and Robert R. Alfano ${ }^{\mathrm{a}, *}$ \\ ${ }^{a}$ The City College of the City University of New York, Institute of Ultrafast Spectroscopy and Lasers, Departments of Physics and Electrical \\ Engineering, 160 Convent Avenue, Room MR 201, New York, New York 10031-9101, United States \\ ${ }^{\text {b}}$ The City University of New York, Borough of Manhattan Community College, 199 Chambers Street, N682, New York, New York 10007-1097, \\ United States \\ 'Energy Research Company, 1250 South Avenue, Plainfield, New Jersey 07062, United States \\ ${ }^{d}$ Columbia University Medical Center, Department of Biomedical Informatics, 622 West 168th Street, PH20, New York, New York 10032, \\ United States \\ ${ }^{\text {e}}$ Columbia University, Department of Chemistry, 3000 Broadway MC 3139, New York, New York 10027, United States
}

\begin{abstract}
A clear correlation has been observed between the resonance Raman (RR) spectra of plaques in the aortic tunica intimal wall of a human corpse and three states of plaque evolution: fibrolipid plaques, calcified and ossified plaques, and vulnerable atherosclerotic plaques (VPs). These three states of atherosclerotic plaque lesions demonstrated unique RR molecular fingerprints from key molecules, rendering their spectra unique with respect to one another. The vibrational modes of lipids, cholesterol, carotenoids, tryptophan and heme proteins, the amide I, II, III bands, and methyl/methylene groups from the intrinsic atherosclerotic VPs in tissues were studied. The salient outcome of the investigation was demonstrating the correlation between RR measurements of VPs and the thickness measurements of fibrous caps on VPs using standard histopathology methods, an important metric in evaluating the stability of a VP. The RR results show that VPs undergo a structural change when their caps thin to $66 \mu \mathrm{m}$, very close to the $65-\mu \mathrm{m}$ empirical medical definition of a thin cap fibroatheroma plaque, the most unstable type of VP. $\odot$ The Authors. Published by SPIE under a Creative Commons Attribution 3.0 Unported License. Distribution or reproduction of this work in whole or in part requires full attribution of the original publication, including its DOI. [DOI: 10.1117/1.JBO.21.12 .127006]
\end{abstract}

Keywords: human artery/aorta; heme protein; methyl/methylene group; molecular fingerprints of amide II and carotenoids; visible resonance Raman spectroscopy using 532-nm excitation; vulnerable atherosclerotic plaque.

Paper 160655RR received Oct. 7, 2016; accepted for publication Nov. 28, 2016; published online Dec. 21, 2016.

\section{Introduction}

Cardiovascular disease is a major cause of morbidity in developed Western countries. Vulnerable atherosclerotic plaque (VP) is an important topic in cardiology research, since patients with these unstable plaques are at great risk for a sudden heart attack (i.e., myocardial infarction) when a plaque suddenly ruptures. Rupture of the thin fibrous cap when its thickness is $<65 \mu \mathrm{m}$, and the resulting subsequent thrombotic occlusion, is the most common cause of death from vulnerable plaque. Finding criteria to identify and measure the degree of risk from these lesions is an active area of investigation. ${ }^{1-6}$ Studies have also shown that a high degree of vulnerable plaque atherosclerosis is a significant indicator of coronary artery disease. ${ }^{7-9}$ Clinically, there are several diagnostic tools, e.g., x-ray angiography, angioscopy, nuclear scintigraphy, and magnetic resonance imaging that are used for identifying the luminal diameter of the aorta and arterial stenosis. However, there are only a few studies that have been performed using Raman spectroscopy and the vibrational frequencies of lipids for detecting atherosclerotic diseases and the thicknesses of cap layers on VPs. ${ }^{10-16}$ None of these studies used resonance Raman (RR).

Optical biopsy techniques, such as label-free native fluorescence, Raman spectroscopy, and optical imaging for in vivo

*Address all correspondence to: Robert R. Alfano, E-mail: ralfano@ccny.cuny .edu cancer detection in human tissues and cells, have been advanced significantly since 1984 by Alfano's group. ${ }^{17-19}$

In our previous studies using 532-nm excitation for RR on human brain, breast, gynecological, gastrointestinal, and atherosclerotic abdominal aortic tissues studies, ${ }^{16,20-24}$ the RR spectra in vitro exhibited native molecular signatures that could be used as optical histopathological criteria to distinguish normal from abnormal tissues. The 532-nm wavelength is a newly important finding for tissue to generate extraordinarily large Raman signals that are useful for quasireal-time measurements. Statistical methods, such as principal component analysis and support vector machine, were used to analyze the RR spectral data collected from benign tumors, cancerous tumors, and normal breast tissues, as well as from meningiomas, benign tumors, and normal meningeal brain tissues. These methods yielded a diagnostic sensitivity of $90.9 \%$ and a specificity of $100 \% .^{20-21}$

Reports detailing the use of the RR technique for studying cardiovascular disease are very limited. One early report on RR scattering spectra in human breast and lung tissues was published by Alfano et al. ${ }^{25}$ for the first time. RR scattering spectra for cardiovascular tissue were first reported by Clarke et al. ${ }^{10} \mathrm{In}$ this work, the authors observed strong RR features from the calcific deposits within the coronary artery and in the aortic valve. They showed a typical Raman spectrum from a fatty plaque within the human coronary artery obtained with a 514.5-nm laser excitation. Three strong peaks dominated the RR spectra at 1006,1156 , and $1517 \mathrm{~cm}^{-1} .{ }^{11}$ The results of other studies on 
the near-infrared (NIR) and Fourier-transform (NIR-FT) Raman spectroscopy by Rava et al. ${ }^{12}$ and by Feld's group ${ }^{13,14,26,27}$ have been used to identify and evaluate human atherosclerotic and vulnerable plaque lesions. These studies described a morphological model for the simultaneous changes in biochemical components, which provided information on different stages of arterial lesions. However, those results were only in the lower frequency spectral region of 750 to $1800 \mathrm{~cm}^{-1} .12-15,26,27$

This report focuses on the visible RR (VRR) technique for directly distinguishing and classifying vulnerable plaques with various states of atherosclerosis development and different cap thicknesses in human aorta tissues using RR spectral molecular fingerprints in a wide frequency region.

\section{Methods and Materials}

\subsection{Methods}

All RR spectra were collected directly from a region of interest along multiple sites on each specimen. The original RR spectrographs, without subtracting for the baseline of light, were produced using WITec Project 2.10 and ORIGIN 2015 software. The Raman spectra were collected from the four specimens shown in Fig. 1, referred to as FAT, M1, M2, and R1. The numbers superimposed on the specimens indicate the sites where spectra were collected and the fibrous cap thicknesses were examined by standard histopathology (Figs. 1 and 2). The histopathology analysis indicated that arteries had extensive calcification and ossification. Thus measurements were made from the edge of the fibrous cap to the beginning of the calcification. Each sample was placed on the stage of the WITec alpha 300R microconfocal Raman and imaging system as has been described in detail previously. ${ }^{16}$ The final spectral resolution was $2 \mathrm{~cm}^{-1}$ in the range of 400 to $3800 \mathrm{~cm}^{-1}$.

\subsection{Human Tissue Sample}

RR spectra of human aorta samples with different states of tissues, including normal fat from the adventitial side arterial wall and three pieces of atherosclerotic plaques lesions, were recorded using a confocal micro-Raman spectrometer. Thirty nine RR spectra were collected from four aortic samples and analyzed. In the RR spectra, a small NIR wing was observed at higher frequencies.

The human aorta specimens exhibiting varying calcific deposits of atherosclerotic vulnerable plaques disease were obtained from the National Disease Research Interchange (NDRI, Philadelphia, Pennsylvania). Some of the tissues exhibited extensive calcification and, in some cases, showed ossification.

The experimental procedures were approved by the City College of the City University of New York, Institutional Review Board (IRB) office. The 12-cm long aorta specimen (Fig. 1, "A") was dissected longitudinally and then cut into four irregularly shaped $\sim 3-\mathrm{cm}$ size pieces as shown in Fig. 1 and marked FAT, M1, M2, and R1. The aorta specimen was obtained from a 92-year-old female with hypercholesterolemia who died from respiratory arrest. The patient had a history of hypertension but no history of diabetes. In general, we used about 200 samples of atherosclerotic abdominal aortic tissues and cells. The samples size ranged from microns to centimeters.

\subsection{Pure, High-Density Lipoprotein, and Low-Density Lipoprotein Cholesterols}

Pure (99\%) cholesterol crystal powder, high-density lipoprotein (HDL), and low-density lipoprotein (LDL) from human plasma in liquid form were purchased from the Sigma-Aldrich Company.
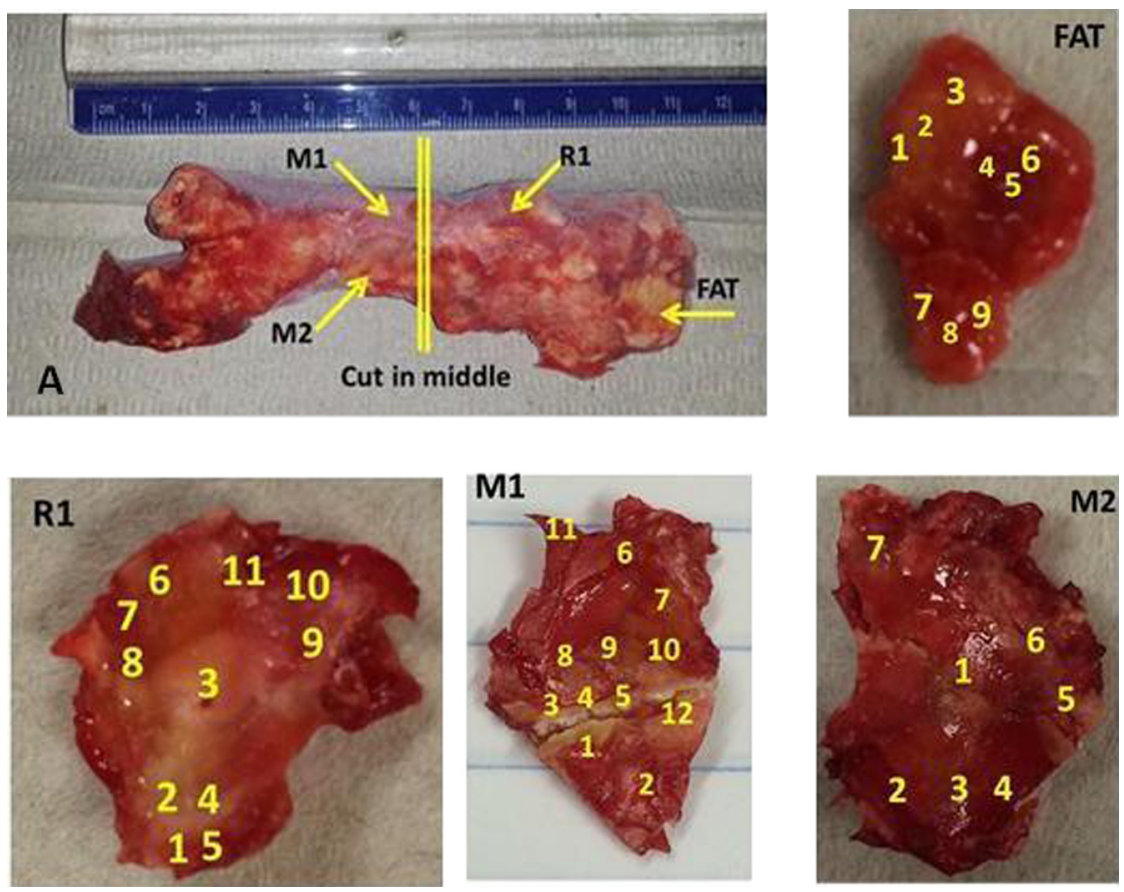

Fig. 1 A photograph of the human aorta tissue specimens used in the experiments. Main section "A" shows the VP tissue obtained from NDRI. Four samples cut from specimen "A" are marked as "FAT", "R1", "M1", and "M2" with the numbers indicating the tested and histopathology examined sites. 


\begin{tabular}{|c|c|c|c|c|}
\hline $\begin{array}{l}\text { Supposed } \\
\text { tissue class }\end{array}$ & RR spectra & $\begin{array}{c}\text { RR site } \\
\text { H\&E Image }\end{array}$ & $\begin{array}{c}\text { Fibrous Cap } \\
\text { Thickness } \\
\text { ( microns) }\end{array}$ & RR site Image \\
\hline $\begin{array}{c}\text { Lipid normal } \\
\text { (yellow class) } \\
\text { (Fig. 3) }\end{array}$ & & & & (d) \\
\hline $\begin{array}{l}\text { Fatty Plaque } \\
\text { [Fig. 4(a)] }\end{array}$ & & (a) & & (e) \\
\hline $\begin{array}{c}\text { Vulnerable } \\
\text { Atherosclerosis } \\
\text { plaque } \\
\text { [Fig. } 4(b)]\end{array}$ & & (b) & 48.68 & \\
\hline $\begin{array}{l}\text { Calcification/ } \\
\text { Ossification } \\
\text { [Fig. 4(c)] }\end{array}$ & & (c) & 74.90 & (g) \\
\hline
\end{tabular}

Fig. 2 Comparisons of normal adventitial arterial wall fat tissue versus three types lesions of atherosclerotic plaques with their RR spectra (column 2), histopathology images (H\&E in column 3), cap thicknesses (column 4), and the confocal microscopy photographs (column 5). (a) The image is the histopathology analysis at the test site M2-07. Vulnerable plaque aorta sample analyzed by Columbia University Medical Center, the measurements are only approximate at the regions marked in Fig. 1, the image covered large areas of the artery. (b) Histopathology image of the test site R103. (c) Histopathology image of the test site R1-01. The RR spectrum from the seriously calcified atherosclerotic plaques and ossification lesion, the feature vibrational mode is a sharp peak at $964 \mathrm{~cm}^{-1}$ with a weaker band at $1076 \mathrm{~cm}^{-1}$. (d) The image is from confocal microscopy photograph at the tested site human artery fat. (e) The image is from confocal microscopy photograph at the tested site M2-07. (f) The image is from confocal microscopy photograph at the tested site R1-03. (g) The image is from confocal microscopy photograph at the tested site R1-01.

\section{Experimental Results and Discussions}

The patterns of RR peaks in the spectra collected from different positions (Fig. 1) on the intimal aortic wall lesions correlate with the disease process as it progresses through the development of arterial fibrolipid plaques, VPs, and calcification and ossification. RR spectra collected from the four laboratory grade samples, including normal fats, chemicals (pure cholesterol powder, HDL, and LDL) (Fig. 3), and three states of atherosclerotic lesions in aortic tissues are shown in Fig. 4.

\subsection{Laboratory Grade Samples}

Figure 3 shows RR spectra from three materials that will help assign peaks in the spectra from the aortic samples (Fig. 4) to specific compounds. Figure 3(a) shows the RR spectrum of pure cholesterol powder, Fig. 3(b) shows the RR spectrum of fat from the tunica adventitial aorta (Fig. 1, "A" marked as FAT). Figure 3 (c) shows the RR spectra of liquid HDL and LDL obtained from human plasma. Figures 3(a)-3(c) highlight the characteristic RR peaks due to cholesterol (located at 704, 1440, and $1674 \mathrm{~cm}^{-1}$ ), to carotenoids (located at 1007,1157 , and $1517 \mathrm{~cm}^{-1}$ ), and to the methylene group $\left[-\mathrm{CH}_{2}-\right]$ vibrational mode and methyl group $\left[-\mathrm{CH}_{3}\right]$ (located at 2854,2895 , and $\left.2939 \mathrm{~cm}^{-1}\right){ }^{28,29}$

\subsection{Fibrolipid Plaques}

The RR spectrum, collected from the sample site numbered M2-07, is shown in Fig. 4(a). Similar RR spectral profiles of M1-02 and R-11 are not shown. A comparison of the spectrum shown in Fig. 4(a) to the RR spectra in Fig. 3 reveals that the Raman peaks common to both spectra are at 1012, 1161, and $1517 \mathrm{~cm}^{-1}$. This suggests an assignment for fatty plaque that is close to that of the lipids in aortic tissue [Figs. 3(b) and 3(c)] and, therefore, is likely to be atherosclerotic aortic tissue. ${ }^{11}$ Lesions in the sites M2-07, M1-02, and R-11 can be considered as representing an early state of the disease process, a noncalcified atherosclerotic fibrolipid plaque whose RR spectrum contains three distinguishable vibrational modes at 1517, 1161, and $1012 \mathrm{~cm}^{-1}$ arising from intense RR lines of carotenoid molecules. These frequencies have been assigned to vibrational modes of the carotene conjugated polyene backbone, and the $1012-\mathrm{cm}^{-1}$ vibrational mode assigned to aromatic amino acids molecules, tryptophan, and phenylalanine. The weak small peak 

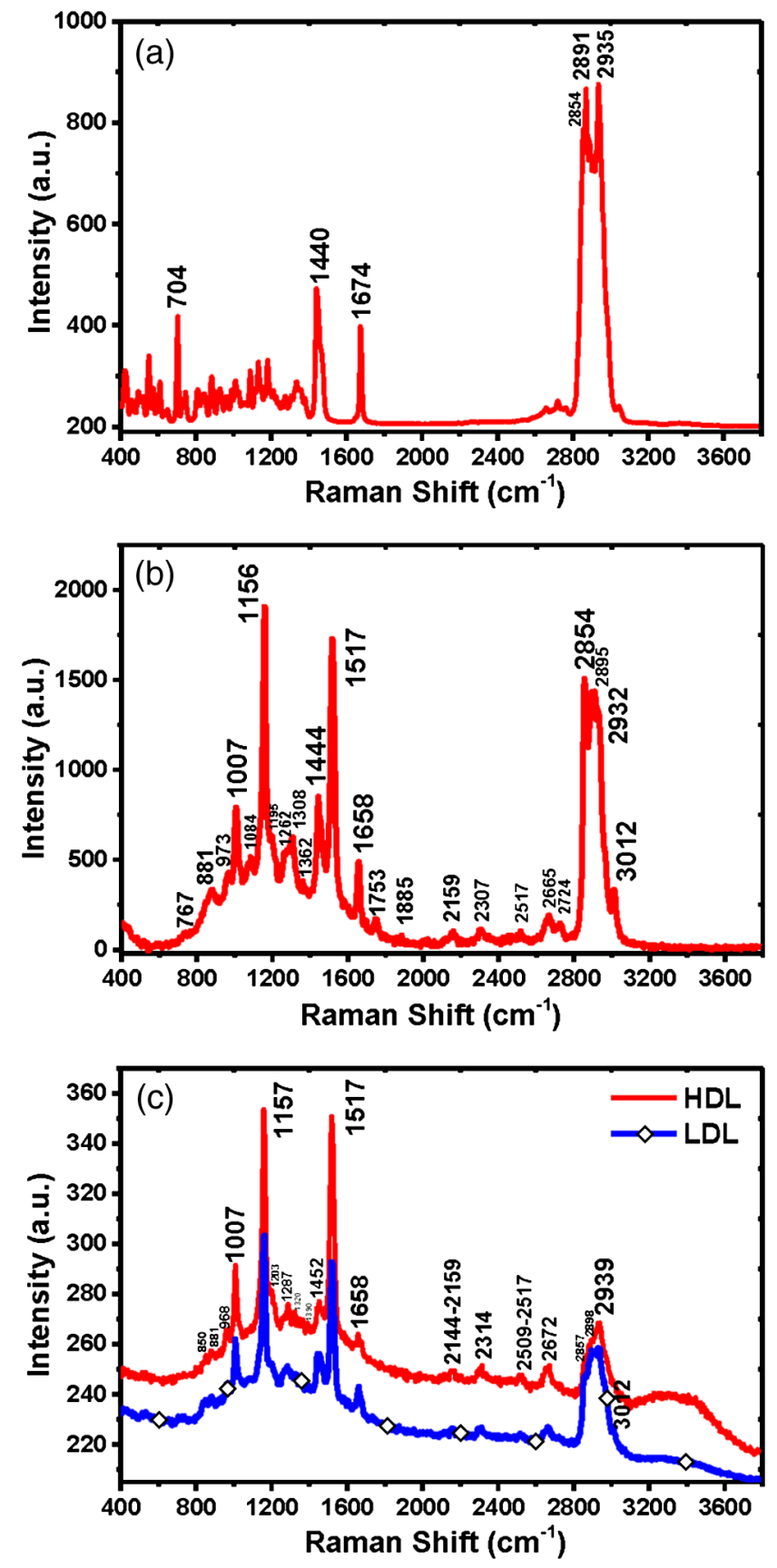

Fig. 3 Typical RR spectra of cholesterol powder, human fat, and lipoproteins in the collected spectral region from 400 to $3800 \mathrm{~cm}^{-1}$, using WITec-300R confocal micro-Raman system with an excitation wavelength of $532 \mathrm{~nm}$ are shown in (a), (b), and (c), respectively. (a) Spectrum is from pure cholesterol powder showing feature peaks at 704, 1440, 1674, 2854, 2891, and $2935 \mathrm{~cm}^{-1}$, (b) spectrum is from human fat showed in Fig. 1 ("A") obtained from normal part of human adventitial arterial wall tissue, characteristic peaks at 1007 $1156,1444,1517,1658,2854,2895$, and $2932 \mathrm{~cm}^{-1}$, (c) spectra are from human plasma of HDL and LDL liquid tissues, substantially similar to the fat Raman peaks, but intense lipids peaks at 1444,1658 , 2854,2895 , and $2932 \mathrm{~cm}^{-1}$ of HDL are obviously weaker than LDL. Those peaks are greatly decreased compared to the fat tissue's intensity peaks

occurring at $964 \mathrm{~cm}^{-1}$ can be attributed to the initial calcification of early stage plaque lesions.

In these lesion sites, a second set of Raman spectral molecular fingerprints arises from the macromolecules of lipids and
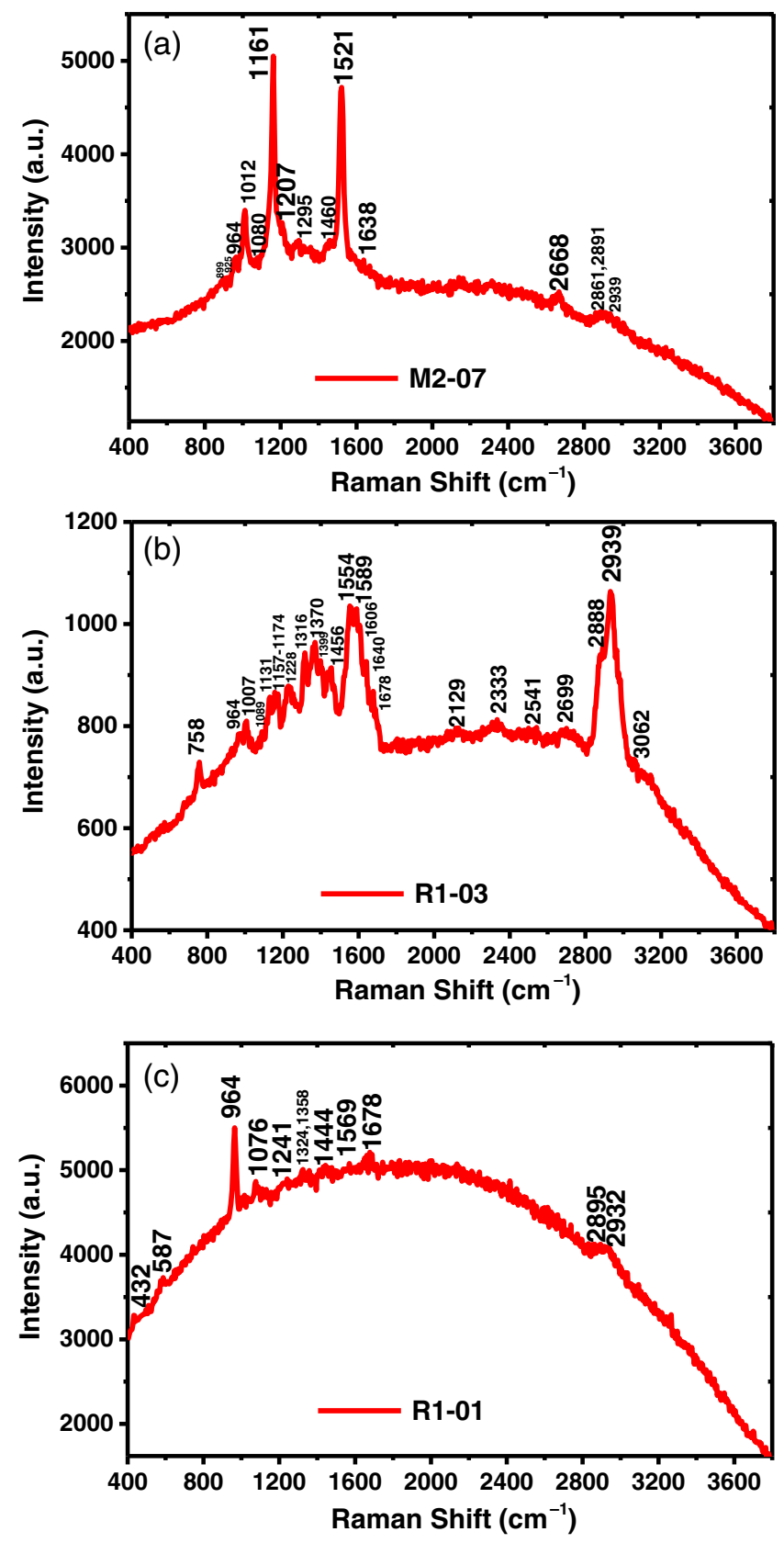

Fig. 4 RR spectral profile variations were recorded from the intimal aortic wall lesions correlated with the different states. (a) A typical RR spectrum of sample M2-07 corresponds to fatty plaque lesion. Three resonance-enhanced peaks at 1012, 1161, and $1521 \mathrm{~cm}^{-1}$, and a vague weak peak at $964 \mathrm{~cm}^{-1}$ were observed, which indicate that early or original calcification. The lipid rich Raman peak at $2854 \mathrm{~cm}^{-1}$ is diminished, and the 2895 and $2932 \mathrm{~cm}^{-1}$ peaks are greatly decreased. (b). Typical RR spectrum of a ruptured atherosclerotic plaque state obtained from lesion site R1-03. The distinct lipid Raman peaks at 2888, 1678, and $1456 \mathrm{~cm}^{-1}$; enhanced protein RR fingerprints at amide II $\left(1554 \mathrm{~cm}^{-1}\right)$, heme protein/tryptophan $\left(1589 \mathrm{~cm}^{-1}\right)$, and lipoprotein $\left(2939 \mathrm{~cm}^{-1}\right)$ reveal a ruptured hemorrhage state lesion. (c). The RR spectrum from the seriously calcified atherosclerotic plaques and ossification lesion. The featured vibrational mode is a sharp peak at $964 \mathrm{~cm}^{-1}$ with a weaker band at $1076 \mathrm{~cm}^{-1}$.

lipoproteins. ${ }^{30}$ These characteristic vibrational modes are at 2854, 2892, and $2934 \mathrm{~cm}^{-1}$, as shown in all three spectra in Fig. 3. Key differences in these peaks are seen in the spectra obtained from normal aortic fat tissue and fibrolipid 
atherosclerotic plaque tissue..$^{11,12,14-16}$ For example, as shown in Figs. 3(a) and 3(b), in HDL/LDL powder and normal aortic fat, the presence of a sharp methylene $\left(-\mathrm{CH}_{2}\right)$ peak is observed at $2854 \mathrm{~cm}^{-1}$. Additionally, the characteristic band of spectral peaks observed at 2932 (Fermi resonance of a $\mathrm{CH}_{3}$ mode) and $2895 \mathrm{~cm}^{-1}$ was attributed to the vibrations of methylene (Fermi resonance of a $-\mathrm{CH}_{2}$ mode) groups. However, in noncalcified atherosclerotic fibrolipid plaque tissue [Fig. 4(a)], the Raman spectral peak at $2854 \mathrm{~cm}^{-1}$ is greatly diminished, while the other two distinct Raman spectral peaks of lipid and lipoproteins at 2895 and $2932 \mathrm{~cm}^{-1}$ decayed greatly. The changes in peak intensity and position in the Raman spectral profiles indicate an important finding. These two characteristics provide a signal to track the transformation of the composition and protein conformation in the cells, as well as the changes in the molecular structure of fibrolipid plaque lesion tissues. ${ }^{31}$

\subsection{Vulnerable Atherosclerotic Plaques}

Figure 4(b) shows a typical spectrum for another type of atherosclerotic plaque, obtained from the R1-03 site (and similar to the RR spectra of R1-04 and M1-01, which are not shown). The three salient characteristics of Raman spectra in this type are: (1) a large lipid pool that has distinct lipid Raman peaks at 2888,1678 , and $1456 \mathrm{~cm}^{-1}$, with a peak at $964 \mathrm{~cm}^{-1}$ that possibly arises from calcified fragments of extracellular debris in ruptured plaque. (2) The enhanced amide III mode at $1228 \mathrm{~cm}^{-1}$ for these type lesions has no shift in its peak position. The sharp intense stable marker at $758 \mathrm{~cm}^{-1}$ can be interpreted as an independent fingerprint. It represents the breathing mode, which gives the most information about the status of red blood cells, as well as direct measures of the heme group in hemoglobin. It is also a characteristic peak of tryptophan, but it is intense and it changes in intensity with the state of atherosclerotic plaques, suggesting that its origin is from heme groups. (3) The intense resonance enhancements of the Raman spectra were displayed in three groups of peaks: (a) one group at 1131 to $1173 \mathrm{~cm}^{-1}$ and a sharp intense peak at $1131 \mathrm{~cm}^{-1}[\nu(\mathrm{C}-\mathrm{C}) \delta(\mathrm{COH})]$ that arose from the fatty acid (i.e., lipid assignment). The significant peak shifts in proteins were observed: $1157 \mathrm{~cm}^{-1}$, at $\nu(\mathrm{C}-\mathrm{C})$, in which the carotenoid was not in normal tissue; $1170 \mathrm{~cm}^{-1}$, where the $\mathrm{C}-\mathrm{H}$ in-plane bending mode of tyrosine (collagen type I) was present; and 1173 and $1174 \mathrm{~cm}^{-1}$, which are the peaks due to tyrosine, phenylalanine, and the $\mathrm{C}-\mathrm{H}$ bend (protein). These spectral profile changes and peak position shifts reflect molecular structure changes corresponding to the difference in fibrous cap thickness. For example, the R1-03 site has a VP cap thickness of $48.68 \mu \mathrm{m}$ and the R1-04 site has a similar thickness of $60.96 \mu \mathrm{m}$, both of which are thinner than the $65-\mu \mathrm{m}$ thickness that defines a highly unstable thin cap fibroatheroma (TCFA) plaque. Data points for both of these samples are included in Fig. 5 and R1-03 is shown in Fig. 2. (b) The second group between 1316 to $1374 \mathrm{~cm}^{-1}$ represents several remarkable modes: $1316 \mathrm{~cm}^{-1}$ depicts the $\mathrm{G}$ (ring breathing modes of the DNA/RNA bases) $-\mathrm{C}-\mathrm{H}$ deformation (protein) and amide III ( $\alpha$-helix), $1370 \mathrm{~cm}^{-1}$ shows the contribution of $\mathrm{T}$, A, G (ring breathing modes of the DNA/RNA, bases-protein), and tryptophan. (c) The third group, between 1554 and $1606 \mathrm{~cm}^{-1}$, contains modes which are marked by the following peaks: the $1554-\mathrm{cm}^{-1}$ peak from the protein amide II, which was derived mainly from the in-plane $\mathrm{N}-\mathrm{H}$ bending, the $1589-\mathrm{cm}^{-1}$ peak indicates the $\mathrm{C}-\mathrm{C}$ stretching and phosphate $(\mathrm{O}-\mathrm{P}-\mathrm{O})$ symmetric stretching vibrational mode, which is a characteristic

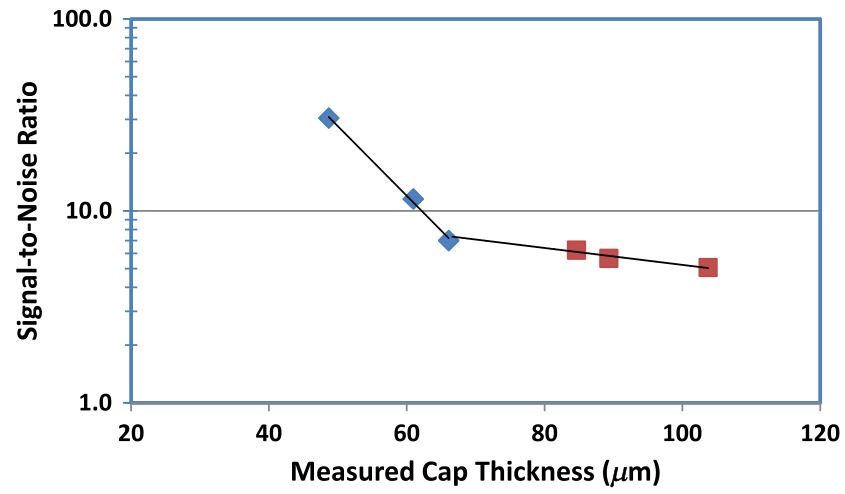

Fig. 5 Intensity changes of the RR peak at the $2895-\mathrm{cm}^{-1}$ vibrational mode plotted as signal-to-noise ratio versus VP cap thickness $(\mu \mathrm{m})$. Two exponential fits to the data are provided illustrating the different trends of Raman signals from smaller cap thickness plaques versus larger cap thickness caps. The two straight lines intercept at $66 \mu \mathrm{m}$, very close to the accepted definition for TCFA of $65 \mu \mathrm{m}$.

of nucleic acids, as well as a known mode for tryptophan, and the $1606-\mathrm{cm}^{-1}$ peak that is due to the $\mathrm{C}-\mathrm{O}$ stretching and $\mathrm{C}-\mathrm{C}$ bending in phenylalanine and tyrosine. ${ }^{32-35}$

These three distinct groups consist mainly of enhanced Raman peaks for proteins, which suggests that the 532-nm excitation wavelength matched (or closely matched) the molecular absorption wavelengths for compounds in the cells and tissues. For example, the metalloprotein, hemoglobin, has one absorption band at $534 \mathrm{~nm}$. Similarly, the mitochondrial electron transport protein, cytochrome $\mathrm{C}$, has one absorption wavelength at $552 \mathrm{~nm}$ (under hypoxia conditions) or the two-photon absorption process under RR conditions that may allow the R1, M1, and M2 sites of the ruptured plaque processes to be seen. ${ }^{32} \mathrm{RR}$ peaks associated with proteins on the molecular level in ruptured fibrolipid plaques tissues were shown and thought to be due to heme proteins, such as the cytochromes that reside in the mitochondria.

These RR spectra from arterial sites may reveal the processes of plaque rupture or thrombosis.

At these positions (R1-03, R1-04, and M1-01), lesions may be classified as advanced in the clinical stage. This is because we found (a) ruptured atherosclerotic plaques with surface erosions, hemorrhages, and thrombus at the test site, as can be seen in the image labeled Fig. 4(b) and column 5 in Fig. 2; (b) the fibrous cap thickness at the tested location on the sample (column 4 of Fig. 2) is a very thin $48.68 \mu \mathrm{m}$, as measured by histopathology; and (c) the RR spectrum at tested site R1-03 in Fig. 5 showed enhanced RR peaks from collagen and fibro muscular tissue layers covering the extracellular lipid pool at 1456, 1554, 1640 , and $1678 \mathrm{~cm}^{-1}$ indicating that the plaque has progressed to the clinically silent and advanced stage. ${ }^{14,26}$

\subsection{Calcified and Ossified Plaques}

Figure 4(c) shows a typical RR spectrum for the third set of lesions where calcification has evolved into ossification. In the highly calcified deposits on the atherosclerotic plaques and ossified lesion, the featured vibrational mode is a sharp peak at $964 \mathrm{~cm}^{-1}$ with a weaker band at $1076 \mathrm{~cm}^{-1}$ that arises from the symmetric stretching vibration of $\nu_{1} \mathrm{PO}_{4}^{3-}$, calciumphosphate stretch band (contains high quantities of cholesterol), and a phosphate symmetric stretching vibration of calcium hydroxyapatite (HA) and quinoid ring in-plane deformation. 
In addition, the $1078-\mathrm{cm}^{-1}$ band arose from the symmetric stretching vibration of $\nu_{1} \mathrm{PO}_{4}^{3-}$ [phosphate of (HA)-single phase $\mathrm{HA}], \nu(\mathrm{C}-\mathrm{C})$ or $\nu(\mathrm{C}-\mathrm{O})$, and phospholipids. In this set of lesions, all the characteristic Raman peaks of lipids and proteins, which appeared in Figs. 4(a) and 4(c), were decayed and diminished. This indicates that, in this state, the main components may be crystalline cholesterol, cholesteryl esters, and phospholipids. $13,15,16,28$

\subsection{Fibrous Cap Thickness}

The RR spectra in different types of atherosclerotic plaque are clearly correlated to the changes of VP fibrous cap thickness as measured by standard histopathology methods and shown in Figs. 2 and 5. The signal-to-noise ratio of the peak intensity at $2895 \mathrm{~cm}^{-1}$ for two classes of plaques varies exponentially with fibrous cap thickness (Fig. 5), but with different exponential decay rates. The samples with small fibrous cap thicknesses exhibit one rate of exponential decay, while those with thicker caps exhibit a much smaller decay rate. This suggest two different cap morphologies for the two types of plaques since the morphology will determine the opacity of the cap to the RR scattered light from the lipid pool and, therefore, the decay rate. The two decay curves intersect near the cap thickness value of $66 \mu \mathrm{m}$. This value is very close to the conventional TCFA definition of $65 \mu \mathrm{m} .{ }^{36,37}$ The result in Fig. 5 is attributed to the transition from ballistic photons propagating through thin caps to diffusive photons propagating through a thicker cap, which occurs at $66 \mu \mathrm{m} .^{38}$

Confirmation of the importance of this finding is left for future work but it suggests that RR measurements of cap thickness can be used to detect the presence of a highly unstable TCFA plaque. Figure 2 shows a comparison of normal arterial fat tissue versus three types lesions of atherosclerotic plaques corresponding to their RR spectra, histopathology images (Fig. 2, column 3), fibrous cap thickness, and the confocal microscopy photographs (Fig. 2, columns 4 and 5). Table 1 lists the vibrational assignments of the modes.

Table 1 Human atherosclerosis aortic specimens obtained from NDRI selected RR spectral peak position and assignment of specimen tissues.

\begin{tabular}{|c|c|c|c|c|}
\hline $\begin{array}{l}\text { Normal fat } \\
\text { (Fig. 3) }\end{array}$ & $\begin{array}{l}\text { Fatty plaque } \\
\text { [Fig. } 4(\mathrm{a})]\end{array}$ & $\begin{array}{l}\text { Ruptured atherosclerosis } \\
\text { plaque [Fig. } 4(\mathrm{~b})]\end{array}$ & $\begin{array}{l}\text { Calcification/ } \\
\text { ossification [Fig. } 4(c)]\end{array}$ & Molecular class/vibration/group \\
\hline 767 vw & $758 w$ & $758 \mathrm{~m}$ & $758 \mathrm{~m}$ & Heme class, trp., C-H out of plane def. \\
\hline $881 \mathrm{w}$ & 872 vw & $833 w$ & - & $\begin{array}{c}\text { C-C, str., cholesterol unesterfied, foam cell necrotic core; } \\
\text { collagen I, fiber and fibrous cap }\end{array}$ \\
\hline 973 vw & $955 \mathrm{w}$ & $920 \mathrm{~m}, 955 \mathrm{w}$ & 964 vs & $\begin{array}{c}\mathrm{PO}_{4}^{3-} \text {, sym/asym, calcium } \mathrm{HA} \text {, phosphate group, } \mathrm{C}-\mathrm{C} \\
\text { backbone (collagen) }\end{array}$ \\
\hline $1007 \mathrm{~s}$ & $1012 \mathrm{~s}$ & $1011 \mathrm{~m}$ & - & Ring breathing, phe \\
\hline $\begin{array}{l}1084 / \\
1072 \mathrm{~m}\end{array}$ & - & $(1078)$ & 1076 w & $\begin{array}{c}\mathrm{C}-\mathrm{C} \text { bending, calcium, cholesteryl esters/carbonate group, } \\
\text { sugar } \mathrm{C}-\mathrm{OH}\end{array}$ \\
\hline 1156 vs & 1161 vs & $1169 \mathrm{~m}$ & - & C-C, str. carotenoids/carotene, collagen type I \\
\hline $1195 \mathrm{sh}$ & - & - & - & C-C, cholesterol \\
\hline $1262 \mathrm{~m}$ & $1207 w$ & $1228 \mathrm{~m}$ & $1241 \mathrm{vw}$ & Amide III enhanced, collagen I \\
\hline $1308 \mathrm{~m}$ & $1316 \mathrm{w}$ & $1316 \mathrm{~m}$ & $1324 \mathrm{vw}$ & $\mathrm{CH}_{2} / \mathrm{CH}_{3}$, bending, triglycerides, collagen \\
\hline 1362 vw & - & $1358 m-1370 w$ & $1358 \mathrm{vw}$ & $-\mathrm{CH}_{3}$ methyl def., $-(\mathrm{C}=\mathrm{O})$ \\
\hline $1444 \mathrm{~s}$ & $1444 \mathrm{~m}$ & $1444 \mathrm{w}$ & $1444 \mathrm{vw}$ & $\mathrm{CH}_{2} / \mathrm{CH}_{3}$, bending, triglycerides, cholesterol \\
\hline 1517 vs & 1517 vs & - & - & $\mathrm{C}=\mathrm{C}$, str. $\beta$-carotene/carotenoids \\
\hline- & - & $1554 \mathrm{~s}$ & - & Amide II, enhanced \\
\hline- & $1586 \mathrm{~m}$ & $1586 \mathrm{~s}$ & - & Heme class, trp. Phe \\
\hline $1658 \mathrm{~s}$ & - & - & 1678 vw & Amide I, protein \\
\hline $1753 \mathrm{~m}$ & - & $1701 \mathrm{vw}$ & - & $\mathrm{C}=\mathrm{O}$ str, cholesterol ester \\
\hline 2854 vs & - & - & - & Lipids \\
\hline $2895 \mathrm{~s}$ & $2892 \mathrm{vw}$ & $2892 \mathrm{~s}$ & $2895 \mathrm{vw}$ & Lipids, $-\mathrm{CH}_{2}-$ \\
\hline $2932 \mathrm{~s}$ & 2939 vw & 2939 vs & 2932 vw & Protein, $-\mathrm{CH}_{3}-$ \\
\hline
\end{tabular}

Note: s, strong; vs, very strong; w, weak; vw, very weak; and $\mathrm{m}$, medium. 


\section{Conclusion}

Common Raman system detection techniques usually cannot generate enhanced resonance molecular Raman lines and high resolution. In this study, e.g., the protein line in the amide II active line at $1554 \mathrm{~cm}^{-1}$, carotene lines at 1161 and $1521 \mathrm{~cm}^{-1}$, amino acid tryptophan lines at 1589 and $758 \mathrm{~cm}^{-1}$, and the intense resonance enhancement in the three groups of peaks [shown in Fig. 4(b)] suggest that the 532-nm excitation wavelength matched (or closely matched) the absorption wavelengths for molecular compounds in the cells and tissues. The VRR system also has the advantage of requiring less power and accumulation time to collect signals, compared with NIR or FT-Raman systems. ${ }^{12-15}$

In conclusion, we have successfully demonstrated that the changes in components and conformation of three states of VPs identified using RR molecular fingerprints and the $2895-\mathrm{cm}^{-1}$ vibrational mode can be detected and identified for lipids that are under the thin intimal wall of the plaque's cap region. The RR spectral findings revealed that these molecular fingerprints can identify the vascular calcification process of atherosclerosis and may provide higher accuracy and sensitivity.

The outcomes of these research findings are:

I. The criteria for detecting the states of VP in fibrous lipid plaque lesions, advanced lesions with calcification to the evolution of ossification, and advanced lesions with ruptured atherosclerotic fibrolipid plaques have been established using the RR spectroscopy technique. The criteria are based on the RR spectra of key natural molecular fingerprints to distinguish changes in lesions. The typical results of Raman spectral molecular fingerprint assessments used to classify the status of atherosclerotic plaque were demonstrated in this study and summarized in Fig. 2 and Table 1. ${ }^{12,20,21,24,39}$ The significant RR molecular markers in different status lesions revealed good agreement between prior work and this study. ${ }^{11,16}$

II. The preliminary analysis of data from the Raman spectral molecular fingerprint at $2895-\mathrm{cm}^{-1}$ mode of cholesterol with various fibrolipid plaque status versus fibrous cap thickness was shown in Fig. 5. The results indicate that the threshold of a critical change in the fibrous cap layer thickness is at $\sim 66 \mu \mathrm{m}$. This finding agrees with the literature on unstable TCFA plaques. $^{36,37}$

\section{Disclosures}

The authors report grants from National Institutes of Health $(\mathrm{NIH})$, during the conduct of the study. In addition, the authors have a patent "Raman and Resonant Raman Detection of Vulnerable Plague Optical Analyzer and Imager" pending.

\section{Acknowledgments}

This work was supported in part by Energy Research Co. from $\mathrm{NIH}$ (National Heart, Lung, and Blood Institute of the NIH under Award No. R41HL126568). The content is solely the responsibility of the authors and does not necessarily represent the official views of the NIH. The authors are grateful to Dr. K. Sutkus and Dr. Lin Zhang for the assistance with paper preparation and submission. Use of human subjects and animals:
In this study, the human aorta specimens were obtained from the NDRI, Philadelphia, Pennsylvania. The experimental procedures were approved by the City College of the City University of New York, IRB office.

\section{References}

1. Z. A. Fayad and V. Fuster, "Clinical imaging of the high-risk or vulnerable atherosclerotic plaque," Circ. Res. 89, 305-316 (2001).

2. L. C. Hofbauer et al., "Vascular calcification and osteoporosis-from clinical observation towards molecular understanding," Osteoporosis Int. 18, 251-259 (2007).

3. M. R. Hayden et al., "Vascular ossification-calcification in metabolic syndrome, type 2 diabetes mellitus, chronic kidney disease, and calciphylaxis-calcific uremic arteriolopathy: the emerging role of sodium thiosulfate," Cardiovasc. Diabetol. 4, 4 (2005).

4. G. A. Rodríguez-Granillo et al., "New insights towards catheter-based identification of vulnerable plaque," Rev. Esp. Cardiol. 58, 1197-1206 (2005).

5. G. P. Fazio et al., "Transesophageal echocardiographically detected atherosclerotic aortic plaque is a marker for coronary artery disease," J. Am. Coll. Cardiol. 21, 144-150 (1993).

6. E. R. Mohler et al., "Bone formation and inflammation in cardiac valves," Circulation 103, 1522-1528 (2001).

7. A. C. van der Wal et al., "Atherosclerotic plaque rupture-pathologic basis of plaque stability and instability," Cardiovasc. Res. 41, 334-344 (1999).

8. B. D. MacNeill et al., "Intravascular modalities for detection of vulnerable plaque: current status," Arterioscler. Thromb. Vasc. Biol. 23, 1333-1342 (2003).

9. S. W. E. van de Poll et al., "On-line detection of cholesterol and calcification by catheter based Raman spectroscopy in human atherosclerotic plaque ex vivo," Heart 89, 1078-1082 (2003).

10. R. H. Clarke et al., "Laser Raman spectroscopy of calcified atherosclerotic lesions in cardiovascular tissue," Appl. Opt. 26, 3175 (1987).

11. R. H. Clarke, Q. Wang, and J. M. Isner, "Laser Raman spectroscopy of atherosclerotic lesions in human coronary artery segments," Appl. Opt. 27, 4799 (1988).

12. R. P. Rava, J. J. Baraga, and M. S. Feld, "Near infrared Fourier transform Raman spectroscopy of human artery," Spectrochim. Acta Part A 47, 509-512 (1991).

13. J. J. Baraga, M. S. Feld, and R. P. Rava, "In situ optical histochemistry of human artery using near infrared Fourier transform Raman spectroscopy," Proc. Natl. Acad. Sci. U. S. A. 89, 3473-3477 (1992).

14. J. T. Motz et al., "In vivo Raman spectral pathology of human atherosclerosis and vulnerable plaque," J. Biomed. Opt. 11, 021003 (2006).

15. G. V. Nogueira et al., "Raman spectroscopy study of atherosclerosis in human carotid artery," J. Biomed. Opt. 10, 031117 (2005).

16. C. Liu et al., "Optical pathology study of human abdominal aorta tissues using confocal micro resonance Raman spectroscopy," Proc. SPIE 9703, 97031S (2016).

17. R. Alfano et al., "Laser induced fluorescence spectroscopy from native cancerous and normal tissue," IEEE J. Quantum Electron. 20, 15071511 (1984).

18. M. Jermyn et al., "Intraoperative brain cancer detection with Raman spectroscopy in humans," Sci. Transl. Med. 7, 274 ra19 (2015).

19. M. S. Bergholt et al., "Fiberoptic confocal Raman spectroscopy for realtime in vivo diagnosis of dysplasia in Barrett's esophagus," Gastroenterology 146, 27-32 (2014).

20. Y. Zhou et al., "Human brain cancer studied by resonance Raman spectroscopy," J. Biomed. Opt. 17, 116021 (2012).

21. C.-H. Liu et al., "Resonance Raman and Raman spectroscopy for breast cancer detection," Technol. Cancer Res. Treat. 12, 371-382 (2013).

22. Y. Zhou et al., "Tumor margin detection using optical biopsy techniques," Proc. SPIE 8940, 894014 (2014).

23. Y. Zhou et al., "Brain metastasis detection by resonant raman optical biopsy method," Proc. SPIE 8940, 89400C (2014).

24. Y. Zhou et al., "Resonant Raman spectra of grades of human brain glioma tumors reveal the content of tryptophan by the $1588 \mathrm{~cm}^{-1}$ mode," Proc. SPIE 9318, 931810 (2015). 
25. R. Alfano et al., "Fluorescence spectra from cancerous and normal human breast and lung tissues," IEEE J. Quantum Electron. 23, 1806-1811 (1987).

26. H. P. Buschman et al., "Raman microspectroscopy of human coronary atherosclerosis: biochemical assessment of cellular and extracellular morphologic structures in situ," Cardiovasc. Pathol. 10, 69-82 (2001).

27. T. J. Romer et al., "Histopathology of human coronary atherosclerosis by quantifying its chemical composition with Raman spectroscopy," Circulation 97, 878-885 (1998).

28. O. Mermut et al., "Directions in peptide interfacial science," Biointerphases 1, P5-P11 (2006).

29. R. M. Kubba, S. H. Rida, and A. H. Hanoon, "Vibration frequencies, normal coordinates and IR absorption intensities of 1-; 1, 2-Di-; 1, 3-Diand 1, 2, 3-trimethylene cyclobutane," Z. Naturforsch. A. 60, 411-418 (2005).

30. M. Ji et al., "Rapid, label-free detection of brain tumors with stimulated Raman scattering microscopy," Sci. Transl. Med. 5, 201 ra119 (2013).

31. N. Haspel et al., "Tracing conformational changes in proteins," $B M C$ Struct. Biol. 10, S1 (2010).

32. S. Pripotnev et al., "UV resonance Raman spectroscopy probes the amide II'p band position in short breast milk peptides with antioxidant activity," J. Raman Spectrosc. 42, 2105-2111 (2011).
33. F. Adar and M. Erecinska, "Photoreductive titration of the resonance Raman spectra of cytochrome oxidase in whole mitochondria," Biochemistry 18, 1825-1829 (1979).

34. M. G. F. Sharp et al., "Differential expression of the mitochondrial gene cytochrome oxidase II in benign and malignant breast tissue," J. Pathol. 168, 163-168 (1992).

35. J. M. Steinke and A. P. Shepherd, "Effects of temperature on optical absorbance spectra of oxy-, carboxy-, and deoxyhemoglobin," Clin. Chem. 38, 1360-1364 (1992).

36. A. P. Burke et al., "Coronary risk factors and plaque morphology in men with coronary disease who died suddenly," N. Engl. J. Med. 336, 12761282 (1997).

37. M. Nakano et al., Coronary Heart Disease, pp. 187-197, Springer, New York (2012)

38. L. Wang et al., "Ballistic 2-D imaging through scattering walls using an ultrafast optical Kerr gate," Science 253, 769-771 (1991).

39. Z. Movasaghi, S. Rehman, and I. U. Rehman, "Raman spectroscopy of biological tissues," Appl. Spectrosc. Rev. 42, 493-541 (2007).

Biographies for the authors are not available. 